\title{
Energy and Throughput Tradeoff in Temporal Spectrum Sharing
}

\author{
Sibel Tombaz, Ki Won Sung, and Jens Zander \\ KTH Royal Institute of Technology, Wireless@KTH, Electrum 229, 16440 Stockholm, Sweden \\ Email: sibel@kth.se, sungkw@kth.se, jenz@kth.se
}

\begin{abstract}
It is envisaged that diverse types of short-range wireless systems coexist in shared spectrum in a near future. For low-power systems, throughput and energy efficiency are two design objectives that often conflict with each other. In this paper, we investigate the tradeoff between the throughput and the energy efficiency for a data-hungry but battery-driven lowpower network which opportunistically shares radio spectrum in temporal domain. We provide a mathematical framework that determines the optimum frame lengths for the different objectives, and analyze the tradeoff. To this purpose, we propose an energy consumption model that reflects the characteristics of lowpower transceivers including power consumption at the receiver side. Numerical results show that the optimum frame length for energy efficiency results in significant loss in throughput, and vice versa. This suggests that the transmission duration of the opportunistic network should be chosen depending on the prime system objective.
\end{abstract}

Index Terms-Temporal spectrum sharing, throughput, energy efficiency, sensing, power consumption.

\section{INTRODUCTION}

Wireless data traffic has been increasing exponentially in recent years and the trend is expected to continue in the coming years [1]. It is also predicted that more than $70 \%$ of traffic will be generated in indoor environments [2]. Therefore, we envisage the coexistence of plethora of short-range wireless systems in a small area in a near future. Spectrum sharing [3] is considered to be a means to enable the coexistence. The usage of industrial, scientific and medical (ISM) band can be given as a successful example of open spectrum sharing. Currently, there exist several technologies operating in $2.4 \mathrm{GHz}$ ISM band such as IEEE 802.11, IEEE 802.15.4, and Bluetooth [4], [5].

We expect there will be much more diverse types of shortrange systems coexisting in shared spectrum. In particular, we consider a coexistence scenario of two data-hungry systems with different transmission powers operating close to each other. Although these systems are assumed to have the same access priority, the system of lower power can be easily out-powered by the one with higher power. Thus, the lowpower system can only access the spectrum in an opportunistic manner to protect its own data reception. We also assume that received signal at the high-power receiver due to the lowpower transmitter is always very small, so that they can be neglected.

Existing schemes for the coexistence of WLAN and WPAN type networks, e.g., frequency hopping and adaptive frequency hopping, etc., are of significant practical concern because of their inadequate performance in time-varying interference behavior and highly loaded traffic scenarios [6]-[8]. Thus sharing of the spectrum resource is assumed to be achieved in the time domain where the low-power network exploits OFF periods of the high-power network.

Frame structure, i.e., transmission and sensing durations, is one of important parameters to consider in the design of opportunistic system. The objective of the low-power network is to maximize its throughput since it is considered dataoriented. At the same time, it is imperative to consume minimum energy because the system is usually battery-driven. Thus, the frame length of the low power network should be determined to attain both objectives, which are generally in tradeoff relationship [9].

Optimum design of frame structure for opportunistic network has been extensively investigated in the context of hierarchical spectrum sharing. In [10]-[13], the lengths of transmission and sensing durations are optimized in order to maximize the throughput of secondary network. However, energy efficiency has not been considered in these works. Energy consumption of low-power nodes has been addressed mostly in the field of sensor network, e.g. [14], [15]. The sensors, however, activate on rare occasions with low data rate requirements. Only a limited study can be found which explored the energy efficiency of data-oriented low-power network [16], [17]. Overall, throughput and energy consumption of opportunistic system has not been investigated together, and thus their tradeoff relationship has not been identified yet.

In this paper, we investigate the the tradeoff between energy efficiency and throughput for data-hungry low-power network that shares radio spectrum opportunistically in time domain. We develop a mathematical framework that takes into account the throughput and energy efficiency at the same time. To this purpose, first we propose a simple energy consumption model that well captures the characteristics of low-power transceivers. Our model considers the power consumption at the receiver side, which is often ignored in the literature. Then, the optimum frame lengths are obtained for the different objectives. To investigate the tradeoff, we analyze the relative loss in energy efficiency when the frame structure is optimized for throughput, and vice versa. We also examine the impact of traffic characteristics of the high-power network on the tradeoff. The proposed framework will provide insights into the design of future low-power networks.

The remainder of this paper is organized as follows: Section 


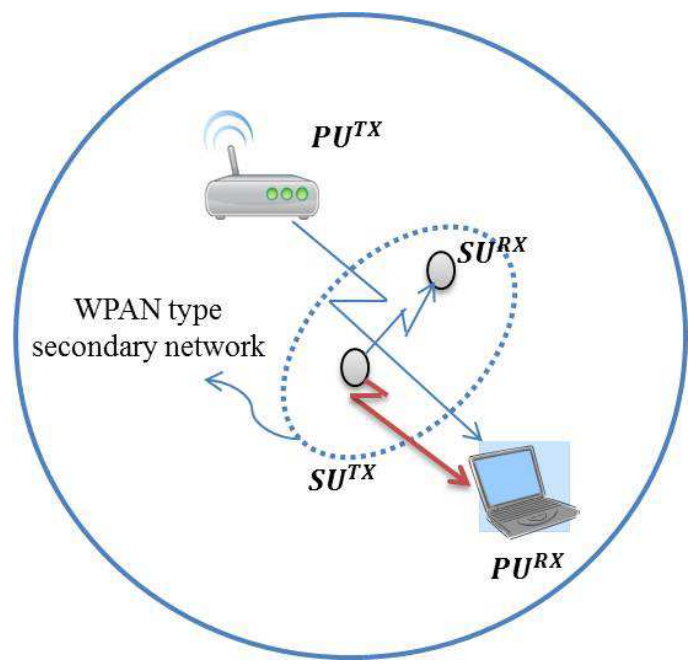

Fig. 1. A deployment scenario for the coexistence of low-power secondary system with primary system in the same geographical area.

II describes the system model we consider and gives detailed problem description. In Section III, we introduce the proposed energy consumption model and provide a mathematical framework to maximize throughput and energy efficiency. Section IV presents the numerical results of tradeoff analysis, and Section V draws the conclusion of the paper.

\section{System Model And Problem Definition}

\section{A. Coexistence Model}

We consider the coexistence of two data-hungry systems with different power levels where each consists of a transmitter and receiver pair located in the same geographical region as shown in Fig. 1. Though these systems are assumed to have the equal right to access the shared spectrum, the lowpower system will need to prevent its transmission from being out-powered by the high-power system. Thus, the low-power system can only access the channel opportunistically where the orthogonality is assumed to be achieved in time domain. To do that, the low-power (secondary) system is required to sense the radio spectrum and detect the state of high-power (primary) node activity. In the case of primary activity detection, the secondary user chooses to stay silent to protect its own data delivery. On the other hand, the primary user is not affected by the secondary user interference due to its relatively high transmission power, and thus does not need to consider the secondary activity.

\section{B. Primary and Secondary Users Activities}

The primary system is assumed to operate in an un-slotted manner and alternates $\mathrm{ON}$ and OFF states according to a stationary Markovian process. ON and OFF periods are assumed to follow exponential distributions with mean durations of $\mathbb{E}\left[T_{O N}\right]=\alpha_{1}$ and $\mathbb{E}\left[T_{O F F}\right]=\alpha_{0}$, respectively. Therefore, probability distribution functions of $\mathrm{ON}$ and OFF periods can be written as follows:

$$
p_{O N}(t)=\frac{1}{\alpha_{1}} e^{-\frac{t}{\alpha_{1}}}, p_{O F F}(t)=\frac{1}{\alpha_{0}} e^{-\frac{t}{\alpha_{0}}} .
$$

The activity of the primary user $\rho$ is defined as

$$
\rho=\frac{\mathbb{E}\left[T_{O N}\right]}{\mathbb{E}\left[T_{O N}\right]+\mathbb{E}\left[T_{O F F}\right]}, \quad \rho \in[0,1) .
$$

On the other hand, the secondary system tries to exploit the temporal opportunities. To do that, it has to sense the channel and determine the state of the frequency band. We consider a fixed-length frame structure of duration $T$ for the secondary user. In each frame, the secondary user first senses the channel in a sensing block with duration $\tau_{S}$ and will transmit or keep silent during the transmission period of $\tau_{T}$ based on the sensing decision $\left(\tau_{S}+\tau_{T}=T\right)$. We assume that the secondary user is heavily loaded and has always data to transmit. Here, we consider a frame structure with the fixed $\tau_{S}$, and aim to optimize $\tau_{T}$.

\section{Energy Consumption Model for Secondary User}

It is essential to capture the comprehensive characteristics of low-power transceivers in the investigation of the secondary system energy efficiency. Power consumption of a transceiver set can be divided into the following modes: silent, transmit, sense, and receive. We let $P_{S}, P_{T}$, and $P_{R}$ denote the power consumed in a unit time for spectrum sensing, data transmission, and reception, respectively. Detailed power consumption elements and their parameter values can be found in [18][20]. It is observed that, in low-power systems, the power for receiving is usually higher than that for transmitting. This allows us to assume that $P_{S}=P_{R}=2 P_{T}$. It is worth emphasizing that the receiving power has not been considered well in the literature.

To calculate the energy consumption of the secondary network, we further make the following assumptions:

- Power consumption of the secondary transceivers in silent mode is negligible [20].

- The secondary receiver is aware of the frame structure, and therefore it stays silent during the sensing periods.

- The secondary receiver tries to receive data at every frame, and stop receiving after $\tau_{S}$ if the secondary activity is not detected.

Energy consumption within a frame differs based on the secondary user's decision on whether to transmit or keep silent. When the channel is detected as vacant, the secondary user will decide to communicate with the energy cost of

$$
E_{T}=P s \tau_{S}+P_{T} \tau_{T}+P_{R} \tau_{T} .
$$

On the other hand, when the activity of the primary user is detected over the channel, the secondary user stays silent. Based on the assumptions made above, consumed energy can be written as

$$
E_{I}=\left(P_{S}+P_{R}\right) \tau_{S}
$$

\section{Problem Formulation}

The design objective of the secondary system is to find the optimum frame length maximizes the throughput by consuming the minimum energy. Therefore, the tradeoff between energy efficiency ( $E E$ : number of successfully delivered bits per 


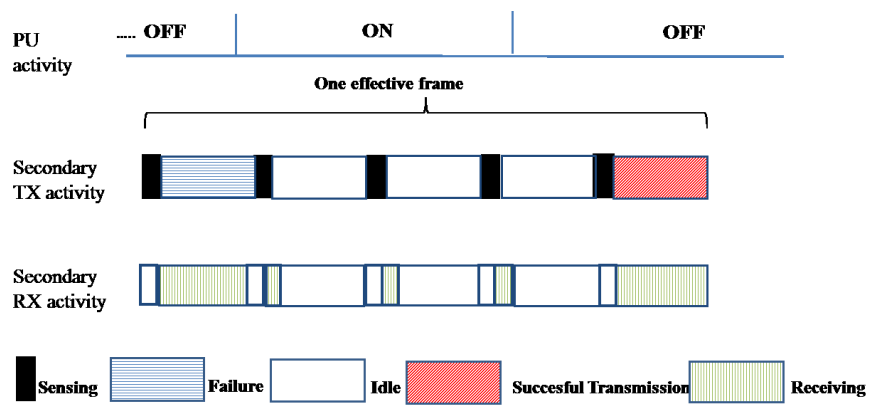

Fig. 2. Activities of primary and secondary users during one effective frame. The primary user switches between $\mathrm{ON}$ and OFF states according to Markovian process and the secondary transmitter and receiver stay idle or communicate accordingly.

joule) and throughput (Th: number of successfully delivered bits per second) has to be investigated. Note that both $E E$ and $T h$ are the functions of $\tau_{T}$. For the performance metrics, the optimization problems can be mathematically expressed as follows:

i) Throughput optimization:

$$
\tau_{T}^{T h}=\underset{\tau_{T}}{\operatorname{argmax}} T h\left(\tau_{T}\right) .
$$

ii) Energy efficiency optimization:

$$
\tau_{T}^{E E}=\underset{\tau_{T}}{\operatorname{argmax}} E E\left(\tau_{T}\right) .
$$

The difference between $\tau_{T}^{T h}$ and $\tau_{T}^{E E}$ leads to the tradeoffs which can be expressed as

$$
\begin{gathered}
\varsigma=\frac{E E\left(\tau_{T}^{E E}\right)-E E\left(\tau_{T}^{T h}\right)}{E E\left(\tau_{T}^{E E}\right)}, \\
\varrho=\frac{T h\left(\tau_{T}^{T h}\right)-T h\left(\tau_{T}^{E E}\right)}{T h\left(\tau_{T}^{T h}\right)},
\end{gathered}
$$

were $\varsigma$ and $\varrho$ represent energy efficiency loss and throughput loss, respectively. These indicate the relative losses in energy efficiency and throughput when $\tau_{T}$ is optimized for the different performance metric.

\section{TRAnsmission Period Optimization AND TRADEOFF ANALYSIS}

In this section, we derive the throughput and the energy efficiency of the secondary network, and demonstrate that there exist unique optimal $\tau_{T}^{T h}$ and $\tau_{T}^{E E}$.

\section{A. Effective Frame}

The secondary user has to spend time and sensing energy while it waits for the vacant channel. Unsuccessful frame due to the sudden activation of the primary user will also cause the waste of the resource. We introduce the concept of effective frame to account for these events. As illustrated in Fig. 2, the effective frame is defined as a group of several frames which consists of the unsuccessful transmission attempts, idle periods, and one successful frame at the end.
The effective frame begins with a transmission attempt of the secondary user. If the primary user activates during the frame, it will cause the loss of the secondary user data, which we call a failure. It is followed by idle frames of awaiting the channel being vacant again. The number of failures and idle frames are denoted by $N_{\text {failure }}$ and $N_{\text {idle }}$, respectively. A successfully delivered frame terminates the effective frame after the possible recurrence of the failures and the idle periods. Therefore, the length of the effective frame $L$ is given by

$$
L=N_{\text {failure }}\left(N_{\text {idle }}+1\right)+1 .
$$

Here, $N_{\text {idle }}$ and $N_{\text {failure }}$ are random variables that can be modeled as geometric distribution with the success probabilities of $q\left(\tau_{T}\right)$ and $p\left(\tau_{T}\right)$, respectively. Since the idle period of the secondary user ends only when the primary user turns to OFF state, $q\left(\tau_{T}\right)$ is as follows:

$$
q\left(\tau_{T}\right)=\int_{0}^{\tau_{T}} p_{O N}(t) d t=\int_{0}^{\tau_{T}} \frac{1}{\alpha_{1}} e^{-\frac{t}{\alpha_{1}}} d t=1-e^{-\frac{\tau_{T}}{\alpha_{1}}}
$$

Thus, the probability mass function of $N_{i d l e}$ is given by

$$
P_{N_{\text {idle }}}(k)=q\left(\tau_{T}\right)\left(1-q\left(\tau_{T}\right)\right)^{k} .
$$

Similarly, $p\left(\tau_{T}\right)$ is represented as below because the failure will not occur when the primary user stays OFF during $\tau_{T}$.

$p\left(\tau_{T}\right)=1-\int_{0}^{\tau_{T}} p_{O F F}(t) d t=1-\int_{0}^{\tau_{T}} \frac{1}{\alpha_{0}} e^{-\frac{t}{\alpha_{0}}} d t=e^{-\frac{\tau_{T}}{\alpha_{0}}}$.

The probability mass function of $N_{\text {failure }}$ is then given by

$$
P_{N_{\text {failure }}}(k)=p\left(\tau_{T}\right)\left(1-p\left(\tau_{T}\right)\right)^{k} .
$$

As it can be seen from (8) and (10), the expectation of the idle frames, $\mathbb{E}\left[N_{\text {idle }}\right]=\frac{1}{q\left(\tau_{T}\right)}$, and the expected value of the failures, $\mathbb{E}\left[N_{\text {failures }}\right]=\frac{1}{p\left(\tau_{T}\right)}$, have the opposite characteristics with regard to the transmission duration $\left(\tau_{T}\right)$. This behavior will affect the energy efficiency and the throughput as will be discussed in the subsequent sections.

\section{B. Throughput}

We assume that a fixed data transmission rate is employed by the secondary user, which is denoted by $r_{T}$. Based on our effective frame definition, a successful transmission only occurs at the last frame of one effective frame. Therefore, the throughput of the secondary user is given by

$$
\mathbb{E}\left[T h\left(\tau_{T}\right)\right]=\frac{r_{T} \tau_{T}}{\mathbb{E}[L]}=\frac{r_{T} \tau_{T}}{\mathbb{E}\left[N_{\text {failure }}\left(N_{\text {idle }}+1\right)+1\right]} .
$$

It is straightforward to show that the throughput is continuously differentiable to $\tau_{T}$. Thus, the the closed form solution of $\tau_{T}^{T h}$ can be obtained by solving $\frac{\partial \mathbb{E}\left[T h\left(\tau_{T}\right)\right]}{\partial \tau_{T}}=0$. 


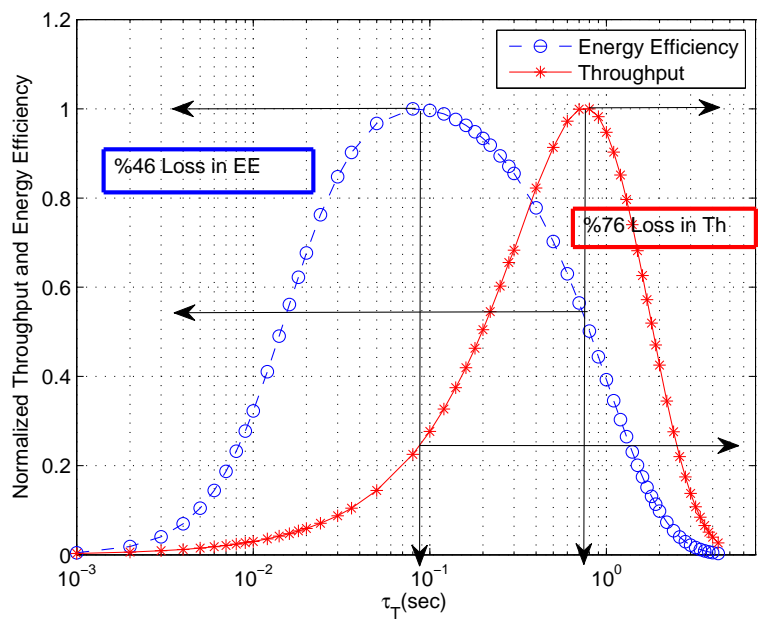

Fig. 3. Achievable normalized throughput and energy efficiency as a function of transmission duration and tradeoff analysis $(\rho=0.35)$

\section{Energy Efficiency}

Let $E_{F}, E_{I}, E_{S}$ denote the energy consumption at a frame of failure, idle, and success, respectively. Then, the total energy consumption during an effective frame $\mathbf{E C}_{t o t}$ can be expressed as

$$
\mathbf{E C}_{\text {tot }}=N_{\text {failure }}\left(E_{F}+N_{\text {idle }} E_{I}\right)+E_{S} .
$$

Note that the energy consumed for the failed frame is equivalent to $E_{S}$, which involves the process of the sensing, transmission, and reception, i.e., $E_{S}=E_{F}=E_{T}$. In the idle frame, the secondary transmitter and receiver are active only during the time of $\tau_{S}$ as explained in (4).

Energy efficiency can be derived by the expected successfully delivered bits and energy consumption during one effective frame. Therefore, the expected value of the $E E\left(\tau_{T}\right)$ is given by (14). The optimal transmission duration for the energy efficiency, $\tau_{T}^{E E}$, can be obtained by the same procedure deriving $\tau_{T}^{T h}$.

\section{NumericAl Results}

In this section, numerical results are presented to demonstrate the tradeoff between the energy efficiency and throughput in terms of the relative energy efficiency loss and throughput loss ( $\varsigma$ and $\varrho$ ) as functions of transmission period $\left(\tau_{T}\right)$. The sensing duration $\tau_{S}$ is fixed to $1 \mathrm{msec}$ throughout the experiments.

Fig. 3 shows the normalized values of energy efficiency and throughput as functions of $\tau_{T}$. The primary user traffic is assumed to be based on VoIP with average ON and OFF durations of $\alpha_{1}=352 \mathrm{msec}$ and $\alpha_{0}=650 \mathrm{msec}$, respectively $(\rho=0.35)$ [21], [22]. Tradeoff between $\tau_{T}^{T h}$ and $\tau_{T}^{E E}$ is clearly noticeable in the figure. It shows that the throughput loss $\varrho$ is $76 \%$ whereas the energy efficiency loss $\varsigma$ is $46 \%$. This suggests that the throughput is more sensitive to the transmission duration $\tau_{T}$.

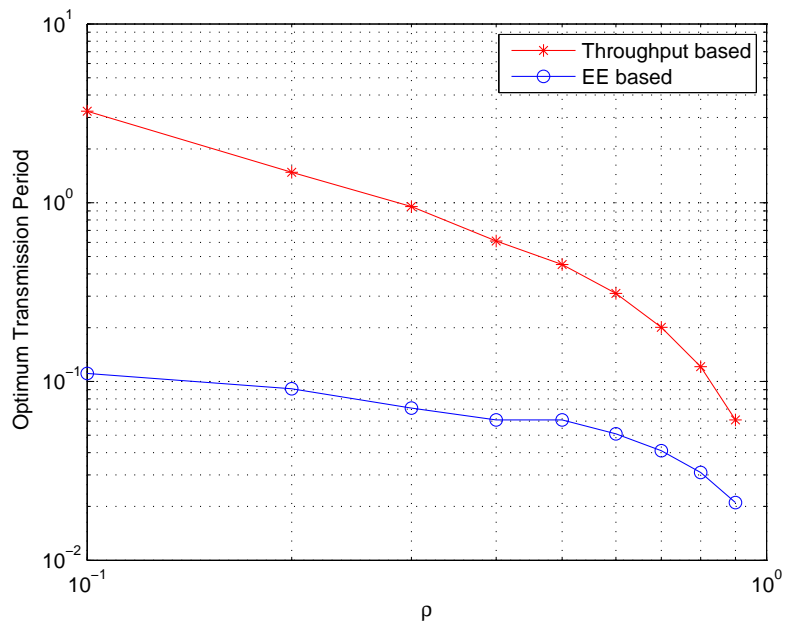

(a) Optimum transmission periods based on different objectives versus activity level.

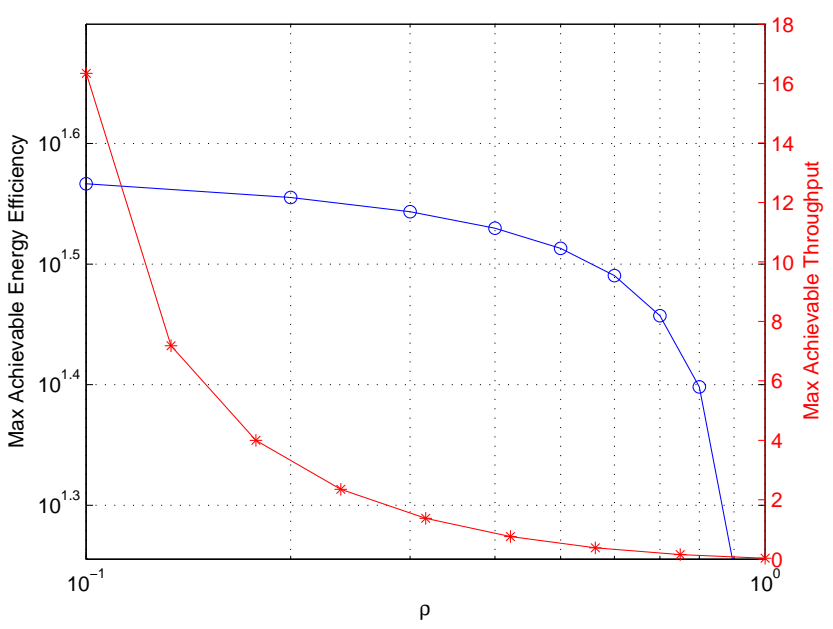

(b) Maximum achievable throughput and energy efficiency versus activity level.

Fig. 4. Impact of primary traffic activity level on energy efficiency and throughput of secondary user $\left(\alpha_{1}=352 \mathrm{msec}\right)$.

Fig. 4(a) and Fig. 4(b) show the effect of the primary traffic activity level on $\tau_{T}^{T h}$ and $\tau_{T}^{E E}$. The mean duration of ON state $\alpha_{1}$ is fixed to $352 \mathrm{msec}$, and the OFF duration $\alpha_{0}$ is varied to account for the different $\rho$ levels, i.e., $\alpha_{0}=\frac{(1-\rho) \alpha_{1}}{\rho}$. The activity level ranges from $\rho=0.1$ to $\rho=0.9$, which captures various modes of the packet voice such as traditional model ( $\rho=0.35)$, conversation $(\rho=0.54)$, and scripted speech $(\rho=0.88)$ [22]. It is observed in Fig. 4(a) that both $\tau_{T}^{T h}$ and $\tau_{T}^{E E}$ decrease as $\rho$ increases. This is because the data delivery of the secondary user is more likely to be interrupted by the activation of the primary user as the the primary traffic gets heavier. The secondary user needs to shorten the transmission duration to avoid the collision between primary and secondary transmissions. Shorter frame length increases the burden of sensing time and energy to the throughput and energy efficiency. Consequently, both metrics decline with higher $\rho$ as depicted in Fig. 4(b). 


$$
\mathbb{E}\left[E E\left(\tau_{T}\right)\right]=\frac{\mathbb{E}\left[T h\left(\tau_{T}\right)\right] \mathbb{E}[L]}{\mathbb{E}\left[\mathbf{E} \mathbf{C}_{\text {tot }}\right]}=\frac{r_{T} \tau_{T}}{\mathbb{E}\left[\left(N_{\text {failure }}+1\right)\left(E_{T}+N_{\text {idle }} E_{I}\right)+E_{T}\right]}
$$

TABLE I

TRADEOFF ANALYSIS FOR DifFERENT ACTIVITY LEVEL $(\rho)$

\begin{tabular}{ccccc}
\hline \hline$\rho$ & $\rho=0.25$ & $\rho=0.35$ & $\rho=0.54$ & $\rho=0.88$ \\
\hline Th Loss $(\varrho)$ & $80.1 \%$ & $76.1 \%$ & $65.4 \%$ & $27.4 \%$ \\
$E E$ Loss $(\varsigma)$ & $46.9 \%$ & $45.8 \%$ & $44.2 \%$ & $43.1 \%$ \\
\hline
\end{tabular}

Table I summarizes the impact of $\rho$ on $\varrho$ and $\varsigma$. When the primary user has a low or moderate channel occupation, $\varrho$ is much higher than $\varsigma$, i.e., the throughput is much more sensitive to a non-optimal transmission duration. On the other hand, for heavy primary traffic, the throughput does not exhibit significant tradeoff. However, the throughput and energy efficiency are already too low in this region as shown in Fig. 4(b), and thus the temporal spectrum sharing does not bring any significant advantage to the secondary user.

\section{COnClusion}

In this paper, we investigated the tradeoff between energy efficiency and throughput for battery-driven low-power network which opportunistically shares the radio spectrum in the temporal domain. We developed a mathematical framework that determines the optimal frame lengths for different objectives. To this purpose, we proposed an energy consumption model that captures the characteristics of low-power transceivers, including the power consumption of the receiver which was missing in the literature. To investigate the tradeoff, we analyzed the relative loss in energy efficiency when the frame is optimized based on throughput, and vice versa. We also examined the impact of the primary user traffic characteristics on the tradeoff.

Numerical results show that there exists a significant difference between the optimal frame lengths which maximize energy efficiency and throughput. It is further outlined that this tradeoff highly depends on the activity level of the primary user. For low and moderate primary user activities where the secondary user can benefit from the temporal spectrum sharing, the energy-based frame length optimization costs considerable loss in the throughput. The loss in energy efficiency caused by the throughput-based frame duration is also severe, but less than the opposite case.

The framework proposed in this work provides insights into the design of future low-power networks. However, only limited scenarios have been examined here. More extensive investigation about the energy and throughput tradeoff should be done as further studies.

\section{REFERENCES}

[1] "Cisco visual networking index: Global mobile data traffic forecast update, 2010-2015," Cisco Inc., Tech. Rep. White paper, February 2011.

[2] T. Norman, "The Road to LTE for GSM and UMTS Operators," Analysis Mason Ltd., Tech. Rep. White paper, January 2009.

[3] J. M. Peha, "Sharing spectrum through spectrum policy reform and cognitive radio," Proc. IEEE, vol. 97, no. 4, pp. 708 -719, April 2009.
[4] C. Chiasserini and R. Rao, "Coexistence mechanisms for interference mitigation in the 2.4-GHz ISM band," IEEE Trans. on Wireless Commun., vol. 2, no. 5, pp. 964 - 975, sept. 2003.

[5] B. P. Crow, I. Widjaja, L. Kim, and P. T. Sakai, "IEEE 802.11 Wireless Local Area Networks," IEEE Commun. Mag., vol. 35, no. 9, pp. 116 -126 , Sep 1997.

[6] S. Lee and Y. H. Lee, "Adaptive frequency hopping for bluetooth robust to WLAN interference," IEEE Commun. Letters, vol. 13, no. 9, pp. 628 -630 , Sept 2009.

[7] L. Stabellini, L. Shi, A. Al Rifai, J. Espino, and V. Magoula, "A new probabilistic approach for adaptive frequency hopping," in Proc. IEEE International Symposium on Personal, Indoor and Mobile Radio Commun.(PIMRC), Sept 2009.

[8] S. Geirhofer, L. Tong, and B. M. Sadler, "Dynamic spectrum access in the time domain: Modeling and exploiting white space," IEEE Commun. Mag., vol. 45, no. 5, pp. $66-72$, May 2007.

[9] S. Tombaz, A. Vastberg, and J. Zander, "Energy- and cost-efficient ultrahigh-capacity wireless access," IEEE Wireless Commun. Mag., vol. 18, no. 5 , pp. $18-24$, October 2011.

[10] Y.-C. Liang, Y. Zeng, E. Peh, and A. T. Hoang, "Sensing-throughput tradeoff for cognitive radio networks," IEEE Trans. on Wireless Commun., vol. 7, no. 4, pp. 1326 -1337, April 2008.

[11] K. W. Sung, S. L. Kim, and J. Zander, "Temporal spectrum sharing based on primary user activity prediction," IEEE Trans. on Wireless Commun., vol. 9, no. 12, pp. $3848-3855$, Dec. 2010.

[12] X. Zhou, Y. Li, Y. H. Kwon, and A. Soong, "Detection timing and channel selection for periodic spectrum sensing in cognitive radio," in Proc. IEEE Global Telecom. Conf. (GLOBECOM), Dec 2008.

[13] Q. Zhao, S. Geirhofer, L. Tong, and B. Sadler, "Opportunistic spectrum access via periodic channel sensing," IEEE Trans. on Signal Process., vol. 56, no. 2, pp. $785-796$, Feb. 2008.

[14] L. Stabellini and J. Zander, "Energy-aware spectrum sensing in cognitive wireless sensor networks: A cross layer approach," in Proc. IEEE Wireless Comm.and Netw. Conf.(WCNC), april 2010.

[15] S. Maleki, A. Pandharipande, and G. Leus, "Energy-efficient distributed spectrum sensing for cognitive sensor networks," IEEE Sensors Journal, vol. 11 , no. 3, pp. $565-573$, March 2011.

[16] L. Li, X. Zhou, H. Xu, G. Li, D. Wang, and A. Soong, "Energyefficient transmission for protection of incumbent users," IEEE Trans. on Broadcasting,, vol. 57, no. 3, pp. 718 -720, Sept. 2011.

[17] A. T. Hoang, Y. C. Liang, D. Wong, R. Zhang, and Y. Zeng, "Opportunistic spectrum access for energy-constrained cognitive radios," in Proc. of IEEE Vehic. Technol. Conf. (VTC Spring), May 2008.

[18] V. Shnayder, M. Hempstead, B. Chen, G. W. Allen, and M. Welsh, "Simulating the power consumption of large-scale sensor network applications," in Proc. ACM Embedded Networked Sensor Systems, 2004.

[19] G. Miao, N. Himayat, G. Li, and A. Swami, "Cross-layer optimization for energy-efficient wireless communications: a survey," Wireless Communications and Mobile Computing, vol. 9, no. 4, pp. 529-542, 2009.

[20] W. Gabran, P. Pawelczak, and D. Cabric, "Multi-channel multi-stage spectrum sensing: Link layer performance and energy consumption," in Proc. IEEE Symposium on Dynamic Spectrum Access Networks (DySPAN), May 2011.

[21] Y. C. Liang, Y. Zeng, E. Peh, and A. T. Hoang, "Sensing-throughput tradeoff for cognitive radio networks," in Proc. IEEE International Conf. on Comm. ( ICC 07), June 2007, pp. $5330-5335$.

[22] S. Deng, "Traffic characteristics of packet voice," in Proc. IEEE International Conf. on Comm.(ICC), vol. 3, jun 1995, pp. 1369 -1374. 